\title{
Stability analysis for a class of discrete schistosomiasis models with general incidence
}

\author{
Aboudramane Guiro ${ }^{1,2 *}$, Diène $\mathrm{Ngom}^{3}$ and Dramane Ouedraogo ${ }^{1,2}$
}

\section{"Correspondence:}

abouguiro@yahoo.fr

${ }^{1}$ Département de Mathématiques,

UFR des Sciences et Techniques,

Bobo-Dioulasso, Burkina Faso

${ }^{2}$ LAboratoire de Mathématique et

d'Informatique (LAMI), Université

Polytechnique de Bobo-Dioulasso,

01 B.P. 1091, Bobo-Dioulasso,

Burkina Faso

Full list of author information is

available at the end of the article

\begin{abstract}
In this paper, we propose to study a class of discrete schistosomiasis models with general incidence function. This model is derived from a continuous schistosomiasis model (in Appl. Math. 4:1682-1693, 2003) by using the backward Euler discretization method with step size $h=1$. We visit some basic properties of this discrete model and we study the stabilities of the equilibria by constructing some appropriate Lyapunov functional for the endemic equilibrium.
\end{abstract}

Keywords: backward Euler method; discrete schistosomiasis models; globally asymptotic stability; Lyapunov functional; general incidence; reproduction number

\section{Introduction}

Schistosomiasis is also known as bilharzia after Theodor Bilharz, who first identified the parasite in Egypt in 1851. This infection is widespread with a relatively low mortality rate but a high morbidity rate, causing severe debilitating illness. It affects populations where substandard conditions of living are predominant, i.e. poor sanitation, insufficient safe water supply and low standard hygiene being practiced [2, 3]. Schistosomiasis remains a public health problem in several parts of the world, particularly in Africa, with more than 240 million people infected and more than 700 million people living in endemic areas [4]. School-age children are most likely to become infected with this silent, destructive disease because it is easily contracted while bathing or swimming in water contaminated with the parasite.

The application of the theory of functional differentials and/or differences in mathematical biology has been developed in the last decades. Many authors have studied the epidemic models, which display the dynamical behavior of the transmission of infected disease. Several mathematical models for schistosomiasis disease have been studied ([1, 5-9]) and the references therein). Most of the models deal with continuous modelization.

Nowadays, there is increasing interest and there are increasingly many research results on discrete epidemic models (see [10-15]. The fact that the epidemiological data are usually collected in discrete time units, such as daily, weekly or monthly, makes the discrete model a natural choice to describe a disease transmission. The result of the discrete model can also be compared with the actual data directly. The discrete model also exhibits a rich dynamical behavior, though it is more complicated than the corresponding continuous

(c) The Author(s) 2017. This article is distributed under the terms of the Creative Commons Attribution 4.0 International License (http://creativecommons.org/licenses/by/4.0/), which permits unrestricted use, distribution, and reproduction in any medium, provided you give appropriate credit to the original author(s) and the source, provide a link to the Creative Commons license, and indicate if changes were made. 
model. However, the study of the discrete epidemic models is comparatively poor due to the complicated expression and difficulty in dynamical analysis.

In this paper, we construct a backward difference scheme for a class of continuous-time schistosomiasis models with general nonlinear incidence derived from [1]. We study the dynamical properties, especially the global asymptotic stability of the disease-free equilibrium and endemic equilibrium for this discrete model. Firstly, we address the basic properties of the model, such as the positivity and the boundedness of solutions. Further, by constructing discrete type Lyapunov functions and using the theory of stability of difference equations, we establish the global asymptotic stability of the equilibria.

The paper is structured as follows. In Section 2, we present the discrete mathematical model derived from the continuous one by using backward Euler discretization. In Section 3, we recall some basic properties of the model. Section 4 is devoted to the equilibrium analysis; thus, we study the global stability of the disease-free equilibrium and the endemic equilibrium. In Section 5, we present the numerical simulation and some comments. Finally, in Section 6, we end the paper by a conclusion.

\section{Discrete mathematical model}

In this section, we derive the discrete schistosomiasis mathematical model. In [1], Traoré et al. studied the stability of the equilibria of the following deterministic continuous-time schistosomiasis model:

$$
\left\{\begin{array}{l}
\frac{d H_{s}}{d t}=b_{h}-d_{h} H_{s}-f\left(S_{i}, H_{s}\right)+\gamma H_{i}, \\
\frac{d H_{i}}{d t}=f\left(S_{i}, H_{s}\right)-d_{h} H_{i}-\gamma H_{i}, \\
\frac{d S_{s}}{d t}=b_{s}-d_{s} S_{s}-g\left(H_{i}, S_{s}\right), \\
\frac{d S_{i}}{d t}=g\left(H_{i}, S_{s}\right)-d_{s} S_{i},
\end{array}\right.
$$

where

$H_{s}(t)$ is the size of the susceptible human population;

$H_{i}(t)$ is the size of the infected human population;

$S_{s}(t)$ is the size of the susceptible snails;

$S_{i}(t)$ is the size of the infected snails;

$b_{h}$ is the recruitment rate of human hosts;

$b_{s}$ is the recruitment rate of snails;

$d_{h}$ is the per capita natural death rate of human hosts;

$d_{s}$ is the per capita natural death rate of snails;

$\gamma$ is the treatment rate of human hosts;

$f$ is the infection function of susceptible human due to infected snails;

$g$ is the infection function of susceptible snails due to infected human.

For the study of system (1), it was assumed that functions $f$ and $g$ satisfy the following assumptions.

H1: $f$ and $g$ are nonnegative $C^{1}$ functions on the nonnegative orthant.

H2: For all $\left(H_{s}, H_{i}, S_{s}, S_{i}\right) \in \mathbb{R}_{+}^{4}, f\left(0, H_{s}\right)=f\left(S_{i}, 0\right)=0$ and $g\left(0, S_{s}\right)=g\left(H_{i}, 0\right)=0$.

H3: For all $\left(H_{s}, H_{i}, S_{s}, S_{i}\right) \in \mathbb{R}_{+}^{4}, f\left(S_{i}, H_{s}\right) \leq f_{1}\left(0, H_{s}^{0}\right) S_{i}$ and $g\left(H_{i}, S_{s}\right) \leq g_{1}\left(0, S_{s}^{0}\right) H_{i}$.

Remark 2.1 Assumption $\mathrm{H} 2$ is a natural assumption which means there is not a new infections when there is not an infectious human or an infectious snail. 
System (1) has a disease-free equilibrium,

$$
E_{\mathrm{DFE}}=\left(H_{s}^{0}, 0, S_{s}^{0}, 0\right)=\left(\frac{b_{h}}{d_{h}}, 0, \frac{b_{s}}{d_{s}}, 0\right)
$$

and an endemic equilibrium $\bar{E}=\left(\bar{H}_{s}, \bar{H}_{i}, \bar{S}_{s}, \bar{S}_{i}\right)$. Also, the basic reproduction number is $R_{0}=\sqrt{\frac{f_{1}\left(0, H_{s}^{0}\right) g_{1}\left(0, S_{s}^{0}\right)}{d_{s}\left(\gamma+d_{h}\right)}}$, where

$$
f_{1}=\frac{\partial f}{\partial S_{i}}\left(S_{i}, H_{s}\right), \quad f_{2}=\frac{\partial f}{\partial H_{s}}\left(S_{i}, H_{s}\right), \quad g_{1}=\frac{\partial g}{\partial H_{i}}\left(H_{i}, S_{s}\right), \quad \text { and } \quad g_{2}=\frac{\partial g}{\partial S_{s}}\left(H_{i}, S_{s}\right)
$$

Remark 2.2 The basic reproduction number evaluates the average number of new infections generated by a single infected individual in a completely susceptible population.

We can summarize the stability results of the equilibria in [1] by the following theorem.

Theorem 2.1 ([1]) The following statement holds.

(i) Let assume $R_{0} \leq 1$. Then the disease-free equilibrium $E_{\mathrm{DFE}}$ of (1) is globally asymptotically stable.

(ii) Let us assume $R_{0}>1$. Then the endemic equilibrium $\bar{E}$ of (1) is globally asymptotically stable.

On the other hand, as we said in our introduction, there occur situations such that constructing discrete epidemic models is a more appropriate approach to understand disease transmission dynamics and to evaluate eradication policies because they permit arbitrary time-step units, preserving the basic features of corresponding continuous-time models. Furthermore, this allows for better use of statistical data for numerical simulations due to the reason that the infection data are compiled at discrete given time intervals. In this paper, motivated by the above facts, we propose the following discrete schistosomiasis models with general incidence, which is derived from system (1) by applying a variation of backward Euler method with a step size $h=1$ :

$$
\left\{\begin{array}{l}
H_{s}(p+1)-H_{s}(p)=b_{h}-d_{h} H_{s}(p+1)-f\left(S_{i}(p+1), H_{s}(p+1)\right)+\gamma H_{i}(p+1), \\
H_{i}(p+1)-H_{i}(p)=f\left(S_{i}(p+1), H_{s}(p+1)\right)-d_{h} H_{i}(p+1)-\gamma H_{i}(p+1), \\
S_{s}(p+1)-S_{s}(p)=b_{s}-d_{s} S_{s}(p+1)-g\left(H_{i}(p+1), S_{s}(p+1)\right), \\
S_{i}(p+1)-S_{i}(p)=g\left(H_{i}(p+1), S_{s}(p+1)\right)-d_{s} S_{i}(p+1),
\end{array}\right.
$$

where $b_{h}, d_{h}, \gamma, b_{s}$ and $d_{s}$ are the same positive constants defined above, similar to the case of continuous-time system (1).

The system (2) always admits a disease-free equilibrium $E_{\mathrm{DFE}}=\left(\frac{b_{h}}{d_{h}}, 0, \frac{b_{s}}{d_{s}}, 0\right)$ and an endemic equilibrium $\bar{E}=\left(\bar{H}_{s}, \bar{H}_{i}, \bar{S}_{s}, \bar{S}_{i}\right)$.

For the global stability of the endemic equilibrium we make the additional assumption: 
H4: For all $\left(H_{s}, H_{i}, S_{s}, S_{i}\right) \in \mathbb{R}^{4}$,

$$
\left\{\begin{array}{l}
1 \leq \frac{\frac{f\left(S_{i}(p+1), H_{S}(p+1)\right)}{H_{S}(p+1)}}{\frac{f\left(\bar{S}_{i}, \bar{H}_{S}\right)}{\bar{H}_{S}}} \leq \min \left\{\frac{S_{i}(p)}{\bar{S}_{i}}, \frac{S_{i}(p+1)}{\bar{S}_{i}}\right\} \\
\text { and } \\
1 \leq \frac{\frac{g\left(H_{i}(p+1), S_{S}(p+1)\right)}{S_{S}(p+1)}}{\frac{g\left(\bar{H}_{i}, \bar{S}_{S}\right)}{\bar{S}_{S}}} \leq \min \left\{\frac{H_{i}(p)}{\bar{H}_{i}}, \frac{H_{i}(p+1)}{\bar{H}_{i}}\right\}
\end{array}\right.
$$

Remark 2.3 Assumption $\mathrm{H} 4$ can be seen as a technical assumption to have $V(p+1)-$ $V(p) \leq 0$. Also, it permits one to ensure the positivity of the variable $H_{s}$ and $S_{s}$.

\section{Basic properties}

The initial conditions of system (2) are

$$
H_{s}(0)>0, \quad H_{i}(0)>0, \quad S_{s}(0)>0 \quad \text { and } \quad S_{i}(0)>0 .
$$

Lemma 3.1 Let $\left(H_{s}(p), H_{i}(p), S_{s}(p), S_{i}(p)\right)$ be a solution of system (2) with initial conditions (4). Then $H_{s}(p)>0, H_{i}(p)>0, S_{s}(p)>0$ and $S_{i}(p)>0$ for any $p>0$.

Proof Considering the second and the fourth equation of system (2), we have

$$
\left\{\begin{array}{l}
H_{i}(p+1)-H_{i}(p)=f\left(S_{i}(p+1), H_{s}(p+1)\right)-d_{h} H_{i}(p+1)-\gamma H_{i}(p+1) \\
S_{i}(p+1)-S_{i}(p)=g\left(H_{i}(p+1), S_{s}(p+1)\right)-d_{s} S_{i}(p+1)
\end{array}\right.
$$

so

$$
\left\{\begin{array}{l}
H_{i}(p+1)=\frac{1}{1+d_{h}+\gamma}\left(f\left(S_{i}(p+1), H_{s}(p+1)\right)+H_{i}(p)\right) \\
S_{i}(p+1)=\frac{1}{1+d_{s}}\left(g\left(H_{i}(p+1), S_{s}(p+1)\right)+S_{i}(p)\right)
\end{array}\right.
$$

thus, $H_{i}(p+1)>0$ and $S_{i}(p+1)>0$.

Considering assumption $\mathrm{H} 4$, we have

$$
\frac{f\left(S_{i}(p+1), H_{s}(p+1)\right)}{H_{s}(p+1)} \geq \frac{f\left(\bar{S}_{i}, \bar{H}_{s}\right)}{\bar{H}_{s}} \text { and } \quad \frac{g\left(H_{i}(p+1), S_{s}(p+1)\right)}{S_{s}(p+1)} \geq \frac{g\left(\bar{H}_{i}, \bar{S}_{s}\right)}{\bar{S}_{s}}
$$

So we conclude that $H_{s}(p+1)>0$ and $S_{s}(p+1)>0$ since $f, g, \bar{H}_{s}$ and $\bar{S}_{s}$ are positive.

Let $H=H_{i}+H_{s}$ and $S=S_{i}+S_{s}$, then system (2) can be written as

$$
\left\{\begin{array}{l}
H(p+1)-H(p)=b_{h}-d_{h} H(p+1) \\
S(p+1)-S(p)=b_{s}-d_{s} S(p+1)
\end{array}\right.
$$

with initial conditions

$$
H(0)>0 \text { and } S(0)>0 \text {. }
$$


Lemma 3.2 Let $(H(p), S(p))$ be the solutions of system (7) with the initial conditions (8). Then $H(p)>0, S(p)>0$ for any $p>0$.

Proof Assume that $H(p)>0, S(p)>0$; then we have

$$
\left\{\begin{array}{l}
\left(1+d_{h}\right) H(p+1)=b_{h}+H(p) \\
\left(1+d_{s}\right) S(p+1)=b_{s}+S(p) .
\end{array}\right.
$$

By the first equation of (9), we have $H(p+1)>0$, and by the second equation of (9), we have $S(p+1)>0$. Hence, by induction, we prove this lemma.

Lemma 3.3 Any solution $(H(p), S(p))$ of system (7) with the initial conditions (8) satisfies $\lim \sup _{p \rightarrow+\infty}(H(p)+S(p))<\frac{b_{h}+b_{s}}{\underline{\mu}}$, where $\underline{\mu}=\min \left\{d_{h}, d_{s}\right\}$.

Proof Let $V(p)=H(p)+S(p)$. From system (7), we have

$$
\begin{aligned}
V(p+1)-V(p) & =b_{h}+b_{s}-\left(d_{h} H(p+1)+d_{s} S(p+1)\right) \\
& \leq b_{h}+b_{s}-\min \left\{d_{h}, d_{s}\right\}(H(p+1)+S(p+1))
\end{aligned}
$$

so

$$
V(p+1)-V(p) \leq b_{h}+b_{s}-\underline{\mu} V(p+1)
$$

from which we have $\lim \sup _{p \rightarrow+\infty} V(p) \leq \frac{b_{h}+b_{s}}{\underline{\mu}}$.

Hence, the proof is complete.

Corollary 3.1 The set

$$
K=\left\{\left(H_{s}, H_{i}, S_{s}, S_{i}\right) \in \mathbb{R}^{4} ; 0 \leq H_{s}+H_{i} \leq \frac{b_{h}}{d_{h}} ; 0 \leq S_{s}+S_{i} \leq \frac{b_{s}}{d_{s}}\right\}
$$

is an invariant and attracting domain for system (2).

\section{Theorem 3.1}

(i) If $R_{0} \leq 1$, then model (2) has only a unique disease-free equilibrium $E_{\mathrm{DFE}}=\left(H_{s}^{0}, 0, S_{s}^{0}, 0\right)$.

(ii) If $R_{0}>1$, then model (2) has unique endemic equilibrium $\bar{E}=\left(\bar{H}_{s}, \bar{H}_{i}, \bar{S}_{s}, \bar{S}_{i}\right)$.

Proof Let $E=\left(H_{s}, H_{i}, S_{s}, S_{i}\right)$ be an equilibrium point of system (2). Using the second and the last equations of (2), we have

$$
\begin{aligned}
& f\left(S_{i}, H_{s}\right)=\left(d_{h}+\gamma\right) H_{i}, \\
& g\left(H_{i}, S_{s}\right)=d_{s} S_{i},
\end{aligned}
$$

so we have

$$
\frac{f\left(S_{i}, H_{s}\right)}{S_{i}} \frac{g\left(H_{i}, S_{s}\right)}{H_{i}}=d_{s}\left(d_{h}+\gamma\right) .
$$


By using system (7), let

$$
\begin{aligned}
& \Phi\left(H_{i}, S_{i}\right)=\frac{f\left(S_{i}, H_{s}^{0}-H_{i}\right)}{S_{i}} \frac{g\left(H_{i}, S_{s}^{0}-S_{i}\right)}{H_{i}}-d_{s}\left(d_{h}+\gamma\right), \\
& \lim _{\left(H_{i}, S_{i}\right) \rightarrow\left(0^{+}, 0^{+}\right)} \Phi\left(H_{i}, S_{i}\right)=\frac{\partial f}{\partial S_{i}}\left(0, H_{s}^{0}\right) \frac{\partial g}{\partial H_{i}}\left(0, S_{s}^{0}\right)-d_{s}\left(d_{h}+\gamma\right)=d_{s}\left(d_{h}+\gamma\right)\left(R_{0}^{2}-1\right),
\end{aligned}
$$

and we have also $\Phi\left(H_{s}^{0}, S_{s}^{0}\right)=-d_{s}\left(d_{h}+\gamma\right)$.

When $R_{0} \leq 1$, we have $\lim _{\left(H_{i}, S_{i}\right) \rightarrow\left(0^{+}, 0^{+}\right)} \Phi\left(H_{i}, S_{i}\right) \leq 0$, thus, there is not any $\left(H_{i}^{*}, S_{i}^{*}\right)>$ $(0,0)$ such that $\Phi\left(H_{s}^{*}, S_{s}^{*}\right)=0$. Therefore system (2) has a unique disease-free equilibrium $E_{\text {DFE. }}$.

When $R_{0}>1$, we have $\lim _{\left(H_{i}, S_{i}\right) \rightarrow\left(0^{+}, 0^{+}\right)} \Phi\left(H_{i}, S_{i}\right) \geq 0$, so there exists $\left(\bar{H}_{i}, \bar{S}_{i}\right) \in\left(0, H_{s}^{0}\right) \times$ $\left(0, S_{s}^{0}\right)$, furthermore $\bar{H}_{s}>0$ and $\bar{S}_{s}>0$. This implies that the system (2) has a unique endemic equilibrium point $\bar{E}=\left(\bar{H}_{s}, \bar{H}_{i}, \bar{S}_{s}, \bar{S}_{i}\right)$.

\section{Equilibria and analysis}

For the stability analysis, we present first the local and global stability of the $E_{\mathrm{DFE}}$ and secondly the global stability of the endemic equilibrium with respect to $R_{0}$.

\subsection{Stability of the disease-free equilibrium $E_{\mathrm{DFE}}$}

Theorem 4.1 If $R_{0} \leq 1$, then

$$
\begin{aligned}
& \lim _{p \rightarrow+\infty} H_{s}(p)=\frac{b_{h}}{d_{h}}, \quad \lim _{p \rightarrow+\infty} H_{i}(p)=0, \\
& \lim _{p \rightarrow+\infty} S_{s}(p)=\frac{b_{s}}{d_{s}} \quad \text { and } \quad \lim _{p \rightarrow+\infty} S_{i}(p)=0,
\end{aligned}
$$

and $E_{\mathrm{DFE}}=\left(\frac{b_{h}}{d_{h}}, 0, \frac{b_{s}}{d_{s}}, 0\right)$ is locally asymptotically stable.

Proof Using the assumption $\mathrm{H} 2$, it follows that $f_{2}\left(0, H_{s}\right)=0$ and $g_{2}\left(0, S_{s}\right)=0$ for all $H_{s}$ and $S_{s}$.

Calculating the linearized system at any point $\left(H_{s}, H_{i}, S_{s}, S_{i}\right)$, we have

$$
\left\{\begin{aligned}
H_{s}(p+1)-H_{s}(p)= & -\left(d_{h}+f_{2}\left(S_{i}(p+1), H_{s}(p+1)\right)\right) H_{s}(p+1) \\
& +\gamma H_{i}(p+1)-f_{1}\left(S_{i}(p+1), H_{s}(p+1)\right) S_{i}(p+1) \\
H_{i}(p+1)-H_{i}(p)= & f_{2}\left(S_{i}(p+1), H_{s}(p+1)\right) H_{s}(p+1)-\left(d_{h}+\gamma\right) H_{i}(p+1) \\
& +f_{1}\left(H_{i}(p+1), S_{s}(p+1)\right) S_{i}(p+1) \\
S_{s}(p+1)-S_{s}(p)= & -g_{1}\left(H_{i}(p+1), S_{s}(p+1)\right) H_{i}(p+1) \\
& -\left(d_{s}+g_{2}\left(H_{i}(p+1), S_{s}(p+1)\right)\right) S_{s}(p+1) \\
S_{i}(p+1)-S_{i}(p)= & g_{1}\left(H_{i}(p+1), S_{s}(p+1)\right) H_{i}(p+1) \\
& \left.+g_{2}\left(H_{i}(p+1), S_{s}(p+1)\right)\right) S_{s}(p+1)-d_{s} S_{i}(p+1)
\end{aligned}\right.
$$

Let $X_{n}=\left(H_{s}(n), H_{i}(n), S_{s}(n), S_{i}(n)\right)$, equation (11) can be rewritten at $E_{\mathrm{DFE}}$ into

$$
X_{p+1}=A^{-1} X_{p}
$$


with

$$
A=\left(\begin{array}{cccc}
1+d_{h} & 0 & 0 & f_{1}\left(0, H_{s}^{0}\right) \\
0 & 1+d_{h}+\gamma & 0 & -f_{1}\left(0, H_{s}^{0}\right) \\
0 & g_{1}\left(0, S_{s}^{0}\right) & 1+d_{s} & 0 \\
0 & -g_{1}\left(0, S_{s}^{0}\right) & 0 & 1+d_{s}
\end{array}\right)
$$

If all the eigenvalues $\sigma$ of matrix $A$ satisfy $|\sigma|>1$, then all the eigenvalues $\lambda$ of the matrix $A^{-1}$ will satisfy $|\lambda|<1$.

The linearization of system (2) at $E_{\mathrm{DFE}}$ gives the following characteristic equation:

$$
\left(1+d_{h}-\lambda\right)\left(1+d_{s}-\lambda\right)\left[\left(1+d_{s}-\lambda\right)\left(1+d_{h}+\gamma-\lambda\right)-f_{1}\left(0, H_{s}^{0}\right) g_{1}\left(0, S_{s}^{0}\right)\right]=0 .
$$

We can see that all solutions $\lambda$ of equation (12) satisfy $|\lambda|>1$. Indeed, equation (12) has roots

$$
\lambda=1+d_{s}>1, \quad \lambda=1+d_{h}>1
$$

and the other roots are given by

$$
\left(1+d_{s}-\lambda\right)\left(1+d_{h}+\gamma-\lambda\right)=f_{1}\left(0, H_{s}^{0}\right) g_{1}\left(0, S_{s}^{0}\right)
$$

Let suppose that there exists at least one root $\lambda_{1}=|\lambda|$ of equation (13) such that $|\lambda|<1$. From the expression of $R_{0}$, we have

$$
f_{1}\left(0, H_{s}^{0}\right) g_{1}\left(0, S_{s}^{0}\right)=R_{0}^{2} d_{s}\left(\gamma+d_{h}\right)
$$

Since $R_{0} \leq 1$, we obtain

$$
\begin{aligned}
& f_{1}\left(0, H_{s}^{0}\right) g_{1}\left(0, S_{s}^{0}\right) \leq d_{s}\left(\gamma+d_{h}\right), \\
& f_{1}\left(0, H_{s}^{0}\right) g_{1}\left(0, S_{s}^{0}\right) \leq d_{s}\left(\gamma+d_{h}\right)<\left(d_{s}+1-\lambda_{1}\right)\left(\gamma+d_{h}+1-\lambda_{1}\right) ;
\end{aligned}
$$

so equation (13) cannot have roots. Hence, $E_{\mathrm{DFE}}$ is locally asymptotically stable according to Theorem 2 in [16].

Now, let us analyze the global behavior of the DFE. The global stability of the DFE will be studied using the basic reproduction number $R_{0}$. We first make the following additional assumption.

Theorem 4.2 The disease-free equilibrium is globally asymptotically stable in $K$ whenever $R_{0} \leq 1$.

Proof The proof is based on using a comparison theorem [17]. By using assumption H3, note that the equations of the infected components in system (2) can be expressed as

$$
\left(\begin{array}{c}
H_{i}(p) \\
S_{i}(p)
\end{array}\right) \geq\left(\begin{array}{cc}
1+d_{h}+\gamma & -f_{1}\left(0, H_{s}^{0}\right) \\
-g_{1}\left(0, S_{s}^{0}\right) & 1+d_{s}
\end{array}\right)\left(\begin{array}{c}
H_{i}(p+1) \\
S_{i}(p+1)
\end{array}\right),
$$


so

$$
\left(\begin{array}{c}
H_{i}(p+1) \\
S_{i}(p+1)
\end{array}\right) \leq B^{-1}\left(\begin{array}{c}
H_{i}(p) \\
S_{i}(p)
\end{array}\right), \quad \text { with } B=\left(\begin{array}{cc}
1+d_{h}+\gamma & -f_{1}\left(0, H_{s}^{0}\right) \\
-g_{1}\left(0, S_{s}^{0}\right) & 1+d_{s}
\end{array}\right)
$$

Using the fact that all the moduli of the eigenvalues of the matrix $B$ are greater than 1 , it follows that the linearized differential inequality system (14) is stable whenever $R_{0} \leq 1$.

Indeed, we can see that all eigenvalues $\lambda$ of the matrix $B$ satisfy equation (13). By the same reasoning as in the proof of theorem 4.1, we deduce that the moduli of the eigenvalues of the matrix $B$ are greater than 1 since $R_{0} \leq 1$.

Consequently, by a standard comparison theorem [17], $\left(H_{i}(p), S_{i}(p)\right) \longrightarrow(0,0)$ as $p \longrightarrow$ $\infty$ for system (14) and substituting $H_{i}=S_{i}=0$ in system (2), we get $H_{s} \longrightarrow H_{s}^{0}$ and $S_{s} \longrightarrow S_{s}^{0}$ as $p \longrightarrow \infty$.

Thus, $\left(H_{s}(p), H_{i}(p), S_{s}(p), S_{i}(p)\right) \longrightarrow\left(H_{s}^{0}, 0, S_{s}^{0}, 0\right)$ as $p \longrightarrow \infty$ for system (2) when $R_{0} \leq 1$. Therefore, $E_{\mathrm{DFE}}$ is globally asymptotically stable if $R_{0} \leq 1$.

\subsection{Stability of the endemic equilibrium $\bar{E}$}

To study the global behavior of this endemic equilibrium $\bar{E}$, we use the assumption $\mathrm{H} 4$, which is the discrete version of the one in [1].

Lemma 4.1 Let $\varphi$ be a $C^{1}$ function on a set $[\min \{a, b\}, \max \{a, b\}]$ with $a, b \in \mathbb{R}_{*}^{+}$and $c \in \mathbb{R}^{+}$. Then

$$
\left(1-c \frac{\ln b-\ln a}{b-a}\right) \leq\left(1-c \min \left\{\frac{1}{a}, \frac{1}{b}\right\}\right)
$$

Proof By using the mean value theorem we get (15).

Theorem 4.3 When $\gamma=0$ and $R_{0}>1$, the endemic equilibrium $\bar{E}$ is globally asymptotically stable in $K$.

Proof If we consider the system (2) when $R_{0}>1$, there exists a unique endemic equilib$\operatorname{rium} \bar{E}$. We now establish the global asymptotic stability of this endemic equilibrium when $\gamma=0$.

Evaluating both sides of (2) at $\bar{E}$ with $\gamma=0$ gives

$$
\left\{\begin{array}{l}
b_{h}=d_{h} \bar{H}_{s}+f\left(\bar{S}_{i}, \bar{H}_{s}\right), \\
d_{h} \bar{H}_{i}=f\left(\bar{S}_{i}, \bar{H}_{s}\right), \\
b_{s}=d_{s} \bar{S}_{s}+g\left(\bar{H}_{i}, \bar{S}_{s}\right), \\
d_{s} \bar{S}_{i}=g\left(\bar{H}_{i}, \bar{S}_{s}\right) .
\end{array}\right.
$$

Let

$$
h(x)=x-1-\ln x,
$$




$$
\begin{aligned}
& V_{h s}(p)=g\left(\bar{H}_{i}, \bar{S}_{s}\right) \bar{H}_{s} h\left(\frac{H_{s}(p)}{\bar{H}_{s}}\right), \\
& V_{h i}(p)=g\left(\bar{H}_{i}, \bar{S}_{s}\right) \bar{H}_{i} h\left(\frac{H_{i}(p)}{\bar{H}_{i}}\right), \\
& V_{s s}(p)=f\left(\bar{S}_{i}, \bar{H}_{s}\right) \bar{S}_{s} h\left(\frac{S_{s}(p)}{\bar{S}_{s}}\right), \\
& V_{s i}(p)=f\left(\bar{S}_{i}, \bar{H}_{s}\right) \bar{S}_{i} h\left(\frac{S_{i}(p)}{\bar{S}_{i}}\right) .
\end{aligned}
$$

We can see that $h: \mathbb{R}_{+}^{*} \rightarrow \mathbb{R}_{+}^{*}$ has the strict global minimum $h(1)=0$. Thus, $V_{h s}(p) \geq 0$, $V_{h i}(p) \geq 0, V_{s s}(p) \geq 0, V_{s i}(p) \geq 0$ with equality if and only if $H_{s}(p)=\bar{H}_{s}, H_{i}(p)=\bar{H}_{i}, S_{s}(p)=$ $\bar{S}_{s}$ and $S_{i}(p)=\bar{S}_{i}$. We will study the behavior of the Lyapunov function

$$
V(p+1)=V_{h s}(p+1)+V_{h i}(p+1)+V_{s s}(p+1)+V_{s i}(p+1) .
$$

We can see that $V(p+1) \geq 0$ with equality if and only if

$$
\frac{H_{s}(p+1)}{\bar{H}_{s}}=1, \quad \frac{H_{i}(p+1)}{\bar{H}_{i}}=1, \quad \frac{S_{s}(p+1)}{\bar{S}_{s}}=1 \quad \text { and } \quad \frac{S_{i}(p+1)}{\bar{S}_{i}}=1 .
$$

The differences $V_{h s}(p+1)-V_{h s}(p), V_{h i}(p+1)-V_{h i}(p), V_{s s}(p+1)-V_{s s}(p)$ and $V_{s i}(p+1)-V_{s i}(p)$ along the solutions of (2) will be calculated separately and then combined to get the desired quantity $V(p+1)-V(p)$.

For this purpose, we use Lemma 4.1 and we suppose that $H_{s}(p) \leq H_{s}(p+1)$; the computation is quite the same as $H_{s}(p+1) \leq H_{s}(p)$. We have

$$
V_{h s}(p+1)-V_{h s}(p)=g\left(\bar{H}_{i}, \bar{S}_{s}\right)\left(1-\bar{H}_{s} \frac{\ln H_{s}(p+1)-\ln H_{s}(p)}{H_{s}(p+1)-H_{s}(p)}\right)\left(H_{s}(p+1)-H_{s}(p)\right) .
$$

By using Lemma 4.1, we have

$$
1-\bar{H}_{s} \frac{\ln H_{s}(p+1)-\ln H_{s}(p)}{H_{s}(p+1)-H_{s}(p)} \leq 1-\frac{\bar{H}_{s}}{H_{s}(p+1)}
$$

and so

$$
V_{h s}(p+1)-V_{h s}(p) \leq g\left(\bar{H}_{i}, \bar{S}_{s}\right)\left(1-\frac{\bar{H}_{s}}{H_{s}(p+1)}\right)\left(H_{s}(p+1)-H_{s}(p)\right) .
$$

Using the first equation of (16) to replace $b_{h}$, we have

$$
\begin{aligned}
V_{h s}(p+1)-V_{h s}(p) \leq & g\left(\bar{H}_{i}, \bar{S}_{s}\right)\left(1-\frac{\bar{H}_{s}}{H_{s}(p+1)}\right)\left(d_{h}\left(\bar{H}_{s}-H_{s}(p+1)\right)\right. \\
& \left.+f\left(\bar{S}_{i}, \bar{H}_{s}\right)-f\left(S_{i}(p+1), H_{s}(p+1)\right)\right) \\
\leq & -d_{h} g\left(\bar{H}_{i}, \bar{S}_{s}\right) \frac{\left(H_{s}(p+1)-\bar{H}_{s}\right)^{2}}{H_{s}(p+1)}+g\left(\bar{H}_{i}, \bar{S}_{s}\right) f\left(\bar{S}_{i}, \bar{H}_{s}\right) \\
& \times\left(1-\frac{f\left(S_{i}(p+1), H_{s}(p+1)\right)}{f\left(\bar{S}_{i}, \bar{H}_{s}\right)}\right)\left(1-\frac{\bar{H}_{s}}{H_{s}(p+1)}\right)
\end{aligned}
$$




$$
\begin{aligned}
\leq & -d_{h} g\left(\bar{H}_{i}, \bar{S}_{s}\right) \frac{\left(H_{s}(p+1)-\bar{H}_{s}\right)^{2}}{H_{s}(p+1)}+g\left(\bar{H}_{i}, \bar{S}_{s}\right) f\left(\bar{S}_{i}, \bar{H}_{s}\right) \\
& \times\left(1-\frac{\bar{H}_{s}}{H_{s}(p+1)}-\frac{f\left(S_{i}(p+1), H_{s}(p+1)\right)}{f\left(\bar{S}_{i}, \bar{H}_{s}\right)}\right. \\
& \left.+\frac{f\left(S_{i}(p+1), H_{s}(p+1)\right)}{f\left(\bar{S}_{i}, \bar{H}_{s}\right)} \frac{\bar{H}_{s}}{H_{s}(p+1)}\right) .
\end{aligned}
$$

By adding and subtracting the quantity $1+\ln \left(\frac{f\left(S_{i}(p+1), H_{s}(p+1)\right)}{f\left(\bar{S}_{i}, \bar{H}_{s}\right)} \frac{\bar{H}_{s}}{H_{s}(p+1)}\right)$, we obtain

$$
\begin{aligned}
V_{h s}(p+1)-V_{h s}(p) \leq & -d_{h} g\left(\bar{H}_{i}, \bar{S}_{s}\right) \frac{\left(H_{s}(p+1)-\bar{H}_{s}\right)^{2}}{H_{s}(p+1)}+g\left(\bar{H}_{i}, \bar{S}_{s}\right) f\left(\bar{S}_{i}, \bar{H}_{s}\right) \\
& \times\left[\left(-\frac{\bar{H}_{s}}{H_{s}(p+1)}+1+\ln \frac{\bar{H}_{s}}{H_{s}(p+1)}\right)\right. \\
& +\left(-\frac{f\left(S_{i}(p+1), H_{s}(p+1)\right)}{f\left(\bar{S}_{i}, \bar{H}_{s}\right)}+1+\ln \frac{f\left(S_{i}(p+1), H_{s}(p+1)\right)}{f\left(\bar{S}_{i}, \bar{H}_{s}\right)}\right) \\
& +\left(\frac{f\left(S_{i}(p+1), H_{s}(p+1)\right)}{f\left(\bar{S}_{i}, \bar{H}_{s}\right)} \frac{\bar{H}_{s}}{H_{s}(p+1)}-1\right. \\
& \left.\left.-\ln \left(\frac{f\left(S_{i}(p+1), H_{s}(p+1)\right)}{f\left(\bar{S}_{i}, \bar{H}_{s}\right)} \frac{\bar{H}_{s}}{H_{s}(p+1)}\right)\right)\right] \\
\leq & -d_{h} g\left(\bar{H}_{i}, \bar{S}_{s}\right) \frac{\left(H_{s}(p+1)-\bar{H}_{s}\right)^{2}}{H_{s}(p+1)}+g\left(\bar{H}_{i}, \bar{S}_{s}\right) f\left(\bar{S}_{i}, \bar{H}_{s}\right) \\
& \times\left[-h\left(\frac{\bar{H}_{s}}{H_{s}(p+1)}\right)-h\left(\frac{f\left(S_{i}(p+1), H_{s}(p+1)\right)}{f\left(\bar{S}_{i}, \bar{H}_{s}\right)}\right)\right. \\
& \left.+h\left(\frac{f\left(S_{i}(p+1), H_{s}(p+1)\right)}{f\left(\bar{S}_{i}, \bar{H}_{s}\right)} \frac{\bar{H}_{s}}{H_{s}(p+1)}\right)\right] .
\end{aligned}
$$

Now, let calculate $V_{h i}(p+1)-V_{h i}(p)$. We use the same technique as above and we assume that $H_{i}(p) \leq H_{i}(p+1)$, so

$$
V_{h i}(p+1)-V_{h i}(p)=g\left(\bar{H}_{i}, \bar{S}_{s}\right)\left(1-\bar{H}_{i} \frac{\ln \left(H_{i}(p+1)\right)-\ln \left(H_{i}(p)\right)}{H_{i}(p+1)-H_{i}(p)}\right)\left(H_{i}(p+1)-H_{i}(p)\right)
$$

and, using Lemma 4.1, we get

$$
\begin{aligned}
V_{h i}(p+1)-V_{h i}(p) \leq & g\left(\bar{H}_{i}, \bar{S}_{s}\right)\left(1-\frac{\bar{H}_{i}}{H_{i}(p+1)}\right)\left(H_{i}(p+1)-H_{i}(p)\right) \\
\leq & g\left(\bar{H}_{i}, \bar{S}_{s}\right)\left(1-\frac{\bar{H}_{i}}{H_{i}(p+1)}\right)\left(f\left(S_{i}(p+1), H_{s}(p+1)\right)-d_{h} H_{i}(p+1)\right) \\
\leq & g\left(\bar{H}_{i}, \bar{S}_{s}\right)\left(1-\frac{\bar{H}_{i}}{H_{i}(p+1)}\right)\left(f\left(\bar{S}_{i}, \bar{H}_{s}\right) \frac{f\left(S_{i}(p+1), H_{s}(p+1)\right)}{f\left(\bar{S}_{i}, \bar{H}_{s}\right)}\right. \\
& \left.-d_{h} \bar{H}_{i} \frac{H_{i}(p+1)}{\bar{H}_{i}}\right) .
\end{aligned}
$$


Using the second equation of (16) to replace $d_{h} \bar{H}_{i}$, we have

$$
\begin{aligned}
V_{h i}(p+1)-V_{h i}(p) \leq & f\left(\bar{S}_{i}, \bar{H}_{s}\right) g\left(\bar{H}_{i}, \bar{S}_{s}\right)\left(1-\frac{\bar{H}_{i}}{H_{i}(p+1)}\right)\left(\frac{f\left(S_{i}(p+1), H_{s}(p+1)\right)}{f\left(\bar{S}_{i}, \bar{H}_{s}\right)}\right. \\
& \left.-\frac{H_{i}(p+1)}{\bar{H}_{i}}\right) \\
\leq & f\left(\bar{S}_{i}, \bar{H}_{s}\right) g\left(\bar{H}_{i}, \bar{S}_{s}\right)\left(1-\frac{H_{i}(p+1)}{\bar{H}_{i}}-\frac{f\left(S_{i}(p+1), H_{s}(p+1)\right)}{f\left(\bar{S}_{i}, \bar{H}_{s}\right)} \frac{\bar{H}_{i}}{H_{i}(p+1)}\right. \\
& \left.+\frac{f\left(S_{i}(p+1), H_{s}(p+1)\right)}{f\left(\bar{S}_{i}, \bar{H}_{s}\right)}\right) .
\end{aligned}
$$

By adding and subtracting the quantity $1+\ln \left(\frac{f\left(S_{i}(p+1), H_{s}(p+1)\right)}{f\left(\bar{S}_{i}, \bar{H}_{s}\right)} \frac{\bar{H}_{i}}{H_{i}(p+1)}\right)$, we obtain

$$
\begin{aligned}
V_{h i}(p+1)-V_{h i}(p) \leq & f\left(\bar{S}_{i}, \bar{H}_{s}\right) g\left(\bar{H}_{i}, \bar{S}_{s}\right)\left[\left(-\frac{H_{i}(p+1)}{\bar{H}_{i}}+1+\ln \frac{H_{i}(p+1)}{\bar{H}_{i}}\right)\right. \\
& +\left(-\frac{f\left(S_{i}(p+1), H_{s}(p+1)\right)}{f\left(\bar{S}_{i}, \bar{H}_{s}\right)} \frac{\bar{H}_{i}}{H_{i}(p+1)}\right. \\
& \left.+1+\ln \left(\frac{f\left(S_{i}(p+1), H_{s}(p+1)\right)}{f\left(\bar{S}_{i}, \bar{H}_{s}\right)} \frac{\bar{H}_{i}}{H_{i}(p+1)}\right)\right) \\
& \left.+\left(\frac{f\left(S_{i}(p+1), H_{s}(p+1)\right)}{f\left(\bar{S}_{i}, \bar{H}_{s}\right)}-1-\ln \frac{f\left(S_{i}(p+1), H_{s}(p+1)\right)}{f\left(\bar{S}_{i}, \bar{H}_{s}\right)}\right)\right] \\
\leq & f\left(\bar{S}_{i}, \bar{H}_{s}\right) g\left(\bar{H}_{i}, \bar{S}_{s}\right)\left[-h\left(\frac{f\left(S_{i}(p+1), H_{s}(p+1)\right)}{f\left(\bar{S}_{i}, \bar{H}_{s}\right)} \frac{\bar{H}_{i}}{H_{i}(p+1)}\right)\right. \\
& \left.-h\left(\frac{H_{i}(p+1)}{\bar{H}_{i}}\right)+h\left(\frac{f\left(S_{i}(p+1), H_{s}(p+1)\right)}{f\left(\bar{S}_{i}, \bar{H}_{s}\right)}\right)\right] .
\end{aligned}
$$

Let now calculate $V_{s s}(p+1)-V_{s s}(p)$ by the same technique. We have

$$
V_{s s}(p+1)-V_{s s}(p)=f\left(\bar{S}_{i}, \bar{H}_{s}\right)\left(1-\bar{S}_{s} \frac{\ln \left(S_{s}(p+1)\right)-\ln \left(S_{s}(p)\right)}{S_{s}(p+1)-S_{s}(p)}\right)\left(S_{s}(p+1)-S_{s}(p)\right) .
$$

Assuming that $S_{s}(p) \leq S_{s}(p+1)$ and using Lemma 4.1, we get

$$
\begin{aligned}
V_{s s}(p+1)-V_{s s}(p) & \leq f\left(\bar{S}_{i}, \bar{H}_{s}\right)\left(1-\frac{\bar{S}_{s}}{S_{s}(p+1)}\right)\left(S_{s}(p+1)-S_{s}(p)\right) \\
& \leq f\left(\bar{S}_{i}, \bar{H}_{s}\right)\left(1-\frac{\bar{S}_{s}}{S_{s}(p+1)}\right)\left(b_{s}-d_{s} S_{s}(p+1)-g\left(H_{i}(p+1), S_{s}(p+1)\right) .\right.
\end{aligned}
$$

Using the third equation of (16) to replace $b_{s}$, we have

$$
\begin{aligned}
V_{s s}(p+1)-V_{s s}(p) \leq & f\left(\bar{S}_{i}, \bar{H}_{s}\right)\left(1-\frac{\bar{S}_{s}}{S_{s}(p+1)}\right)\left(d_{s}\left(\bar{S}_{s}-S_{s}(p+1)\right)\right. \\
& \left.+g\left(\bar{H}_{i}, \bar{S}_{s}\right)-g\left(H_{i}(p+1), S_{s}(p+1)\right)\right) \\
\leq & -d_{s} f\left(\bar{S}_{i}, \bar{H}_{s}\right) \frac{\left(S_{s}(p+1)-\bar{S}_{s}\right)^{2}}{S_{s}(p+1)}+f\left(\bar{S}_{i}, \bar{H}_{s}\right) g\left(\bar{H}_{i}, \bar{S}_{s}\right)
\end{aligned}
$$




$$
\begin{aligned}
& \times\left(1-\frac{g\left(H_{i}(p+1), S_{s}(p+1)\right)}{g\left(\bar{H}_{i}, \bar{S}_{s}\right)}\right)\left(1-\frac{\bar{S}_{s}}{S_{s}(p+1)}\right) \\
\leq & -d_{s} f\left(\bar{S}_{i}, \bar{H}_{s}\right) \frac{\left(S_{s}(p+1)-\bar{S}_{s}\right)^{2}}{S_{s}(p+1)}+f\left(\bar{S}_{i}, \bar{H}_{s}\right) g\left(\bar{H}_{i}, \bar{S}_{s}\right) \\
& \times\left(1-\frac{\bar{S}_{s}}{S_{s}(p+1)}-\frac{g\left(H_{i}(p+1), S_{s}(p+1)\right)}{g\left(\bar{H}_{i}, \bar{S}_{s}\right)}\right. \\
& \left.+\frac{g\left(H_{i}(p+1), S_{s}(p+1)\right)}{g\left(\bar{H}_{i}, \bar{S}_{s}\right)} \frac{\bar{S}_{s}}{S_{s}(p+1)}\right) .
\end{aligned}
$$

By adding and subtracting the quantity $1+\ln \left(\frac{g\left(H_{i}(p+1), S_{S}(p+1)\right)}{g\left(\bar{H}_{i} \bar{S}_{S}\right)} \frac{\bar{S}_{s}}{S_{s}(p+1)}\right)$, we obtain

$$
\begin{aligned}
V_{s s}(p+1)-V_{s s}(p) \leq & -d_{s} f\left(\bar{S}_{i}, \bar{H}_{s}\right) \frac{\left(S_{s}(p+1)-\bar{S}_{s}\right)^{2}}{S_{s}(p+1)}+f\left(\bar{S}_{i}, \bar{H}_{s}\right) g\left(\bar{H}_{i}, \bar{S}_{s}\right) \\
& \times\left[\left(-\frac{\bar{S}_{s}}{S_{s}(p+1)}+1+\ln \frac{\bar{S}_{s}}{S_{s}(p+1)}\right)+\left(-\frac{g\left(H_{i}(p+1), S_{s}(p+1)\right)}{g\left(\bar{H}_{i}, \bar{S}_{s}\right)}\right.\right. \\
& \left.+1+\ln \frac{g\left(H_{i}(p+1), S_{s}(p+1)\right)}{g\left(\bar{H}_{i}, \bar{S}_{s}\right)}\right)+\left(\frac{g\left(H_{i}(p+1), S_{s}(p+1)\right.}{g\left(\bar{H}_{i}, \bar{S}_{s}\right)} \frac{\bar{S}_{s}}{S_{s}(p+1)}\right. \\
& \left.\left.-1-\ln \left(\frac{g\left(H_{i}(p+1), S_{s}(p+1)\right)}{g\left(\bar{H}_{i}, \bar{S}_{s}\right)} \frac{\bar{S}_{s}}{S_{s}(p+1)}\right)\right)\right] \\
\leq & -d_{s} f\left(\bar{S}_{i}, \bar{H}_{s}\right) \frac{\left(S_{s}(p+1)-\bar{S}_{s}\right)^{2}}{S_{s}(p+1)}+f\left(\bar{S}_{i}, \bar{H}_{s}\right) g\left(\bar{H}_{i}, \bar{S}_{s}\right) \\
& \times\left[-h\left(\frac{\bar{S}_{s}}{S_{s}(p+1)}\right)-h\left(\frac{g\left(H_{i}(p+1), S_{s}(p+1)\right.}{g\left(\bar{H}_{i}, \bar{S}_{s}\right)}\right)\right. \\
& \left.+h\left(\frac{g\left(H_{i}(p+1), S_{s}(p+1)\right)}{g\left(\bar{H}_{i}, \bar{S}_{s}\right)} \frac{\bar{S}_{s}}{S_{s}(p+1)}\right)\right] .
\end{aligned}
$$

After that, let us evaluate $V_{s i}(p+1)-V_{s i}(p)$. We have

$$
V_{s i}(p+1)-V_{s i}(p)=f\left(\bar{S}_{i}, \bar{H}_{s}\right)\left(1-\bar{S}_{i} \frac{\ln \left(S_{i}(p+1)\right)-\ln \left(S_{i}(p)\right)}{S_{i}(p+1)-S_{i}(p)}\right)\left(S_{i}(p+1)-S_{i}(p)\right) .
$$

Assuming that $S_{i}(p) \leq S_{i}(p+1)$ and using Lemma 4.1, we get

$$
\begin{aligned}
V_{s i}(p+1)-V_{s i}(p) \leq & f\left(\bar{S}_{i}, \bar{H}_{s}\right)\left(1-\frac{\bar{S}_{i}}{S_{i}(p+1)}\right)\left(S_{i}(p+1)-S_{i}(p)\right) \\
\leq & f\left(\bar{S}_{i}, \bar{H}_{s}\right)\left(1-\frac{\bar{S}_{i}}{S_{i}(p+1)}\right)\left(g\left(H_{i}(p+1), S_{s}(p+1)\right)-d_{s} S_{i}(p+1)\right) \\
\leq & f\left(\bar{S}_{i}, \bar{H}_{s}\right)\left(1-\frac{\bar{S}_{i}}{S_{i}(p+1)}\right)\left(g\left(\bar{H}_{i}, \bar{S}_{s}\right) \frac{g\left(H_{i}(p+1), S_{S}(p+1)\right)}{g\left(\bar{H}_{i}, \bar{S}_{s}\right)}\right. \\
& \left.-d_{s} \bar{S}_{i} \frac{S_{i}(p+1)}{\bar{S}_{i}}\right)
\end{aligned}
$$


Using the last equation of (16) to replace $d_{s} S_{i}(p+1)$, we have

$$
\begin{aligned}
V_{s i}(p+1)-V_{s i}(p) \leq & g\left(\bar{H}_{i}, \bar{S}_{s}\right) f\left(\bar{S}_{i}, \bar{H}_{s}\right)\left(1-\frac{\bar{S}_{i}}{S_{i}(p+1)}\right) \\
& \times\left(\frac{g\left(H_{i}(p+1), S_{s}(p+1)\right)}{g\left(\bar{H}_{i}, \bar{S}_{s}\right)}-\frac{S_{i}(p+1)}{\bar{S}_{i}}\right) \\
\leq & g\left(\bar{H}_{i}, \bar{S}_{s}\right) f\left(\bar{S}_{i}, \bar{H}_{s}\right)\left(1-\frac{S_{i}(p+1)}{\bar{S}_{i}}-\frac{g\left(H_{i}(p+1), S_{s}(p+1)\right)}{g\left(\bar{H}_{i}, \bar{S}_{s}\right)} \frac{\bar{S}_{i}}{S_{i}(p+1)}\right. \\
& \left.+\frac{g\left(H_{i}(p+1), S_{s}(p+1)\right)}{g\left(\bar{H}_{i}, \bar{S}_{s}\right)}\right)
\end{aligned}
$$

By adding and subtracting the quantity $1+\ln \left(\frac{g\left(H_{i}(p+1), S_{s}(p+1)\right)}{g\left(\bar{H}_{i}, \bar{S}_{s}\right)} \frac{\bar{S}_{i}}{S_{i}(p+1)}\right)$, we obtain

$$
\begin{aligned}
V_{s i}(p+1)-V_{s i}(p) \leq & g\left(\bar{H}_{i}, \bar{S}_{s}\right) f\left(\bar{S}_{i}, \bar{H}_{s}\right)\left[\left(-\frac{S_{i}(p+1)}{\bar{S}_{i}}+1+\ln \frac{S_{i}(p+1)}{\bar{S}_{i}}\right)\right. \\
& +\left(-\frac{g\left(H_{i}(p+1), S_{s}(p+1)\right.}{g\left(\bar{H}_{i}, \bar{S}_{s}\right)} \frac{\bar{S}_{i}}{S_{i}(p+1)}\right. \\
& \left.+1+\ln \left(\frac{g\left(H_{i}(p+1), S_{s}(p+1)\right)}{g\left(\bar{H}_{i}, \bar{S}_{s}\right)} \frac{\bar{S}_{i}}{S_{i}(p+1)}\right)\right) \\
& \left.+\left(\frac{g\left(H_{i}(p+1), S_{s}(p+1)\right)}{g\left(\bar{H}_{i}, \bar{S}_{s}\right)}-1-\ln \frac{g\left(H_{i}(p+1), S_{s}(p+1)\right)}{g\left(\bar{H}_{i}, \bar{S}_{s}\right)}\right)\right] \\
\leq & f\left(\bar{S}_{i}, \bar{H}_{s}\right) g\left(\bar{H}_{i}, \bar{S}_{s}\right)\left[-h\left(\frac{S_{i}(p+1)}{\bar{S}_{i}}\right)\right. \\
& -h\left(\frac{g\left(H_{i}(p+1), S_{s}(p+1)\right)}{g\left(\bar{H}_{i}, \bar{S}_{s}\right)} \frac{\bar{S}_{i}}{S_{i}(p+1)}\right) \\
& \left.+h\left(\frac{g\left(H_{i}(p+1), S_{s}(p+1)\right)}{g\left(\bar{H}_{i}, \bar{S}_{s}\right)}\right)\right]
\end{aligned}
$$

Combining the above computations of the differences of $V_{h s}, V_{h i}, V_{s s}$ and $V_{s i}$ along the solutions of (2), let us evaluate finally the difference of $V$. We have

$$
\begin{aligned}
V(p+1)-V(p) \leq & -d_{h} g\left(\bar{H}_{i}, \bar{S}_{s}\right) \frac{\left(H_{s}(p+1)-\bar{H}_{s}\right)^{2}}{H_{s}(p+1)}-d_{s} f\left(\bar{S}_{i}, \bar{H}_{s}\right) \frac{\left(S_{s}(p+1)-\bar{S}_{s}\right)^{2}}{S_{s}(p+1)} \\
& +f\left(\bar{S}_{i}, \bar{H}_{s}\right) g\left(\bar{H}_{i}, \bar{S}_{s}\right)\left[-h\left(\frac{S_{i}(p+1)}{\bar{S}_{i}}\right)\right. \\
& +h\left(\frac{f\left(S_{i}(p+1), H_{s}(p+1)\right)}{f\left(\bar{S}_{i}, \bar{H}_{s}\right)} \frac{\bar{H}_{s}}{H_{s}(p+1)}\right)-h\left(\frac{H_{i}(p+1)}{\bar{H}_{i}}\right) \\
& +h\left(\frac{g\left(H_{i}(p+1), S_{s}(p+1)\right)}{g\left(\bar{H}_{i}, \bar{S}_{s}\right)} \frac{\bar{S}_{s}}{S_{s}(p+1)}\right) \\
& -h\left(\frac{f\left(S_{i}(p+1), H_{s}(p+1)\right)}{f\left(\bar{S}_{i}, \bar{H}_{s}\right)} \frac{\bar{H}_{i}}{H_{i}(p+1)}\right)-h\left(\frac{\bar{S}_{s}}{S_{s}(p+1)}\right) \\
& \left.-h\left(\frac{\bar{H}_{s}}{H_{s}(p+1)}\right)-h\left(\frac{g\left(H_{i}(p+1), S_{s}(p+1)\right)}{g\left(\bar{H}_{i}, \bar{S}_{s}\right)} \frac{\bar{S}_{i}}{S_{i}(p+1)}\right)\right] .
\end{aligned}
$$


Since the function $h$ is monotone on each side of point 1 and is minimized at this point 1 , H4 implies

$$
\begin{aligned}
& h\left(\frac{f\left(S_{i}(p+1), H_{s}(p+1)\right)}{f\left(\bar{S}_{i}, \bar{H}_{s}\right)} \frac{\bar{H}_{s}}{H_{s}(p+1)}\right) \leq h\left(\frac{S_{i}(p+1)}{\bar{S}_{i}}\right) \text { and } \\
& h\left(\frac{g\left(H_{i}(p+1), S_{s}(p+1)\right)}{g\left(\bar{H}_{i}, \bar{S}_{s}\right)} \frac{\bar{S}_{s}}{S_{s}(p+1)}\right) \leq h\left(\frac{H_{i}(p+1)}{\bar{H}_{i}}\right) .
\end{aligned}
$$

Since $h(x) \geq 0 \forall x \in \mathbb{R}$,

$$
V(p+1)-V(p) \leq 0
$$

so for all $\left(H_{s}, H_{i}, S_{s}, S_{i}\right) \in K$ with equality only for $H_{s}(p+1)=\bar{H}_{s}, H_{i}(p+1)=\bar{H}_{i}, S_{s}(p+1)=\bar{S}_{s}$ and $S_{i}(p+1)=\bar{S}_{i}$.

Hence, the endemic equilibrium $\bar{E}$ is the only positively invariant set of the system (2) contained in $\left\{\left(H_{s}, H_{i}, S_{s}, S_{i}\right) \in \mathbb{R}_{+}^{4} ; H_{s}=\bar{H}_{s}, H_{i}=\bar{H}_{i}, S_{s}=\bar{S}_{s}, S_{i}=\bar{S}_{i}\right\}$. Then, by the Lyapunov theorems on the global asymptotical stability for difference equations [18], we see that the endemic equilibrium $\bar{E}$ is globally asymptotically stable. This completes the proof.

\section{Numerical simulation and comments}

In this part, we give some results for the discrete and continuous version on the schistosomiasis model (1). So we perform the computation work that supports our study. In this computation, the functions $f$ and $g$ are chosen as follows: $f\left(S_{i}, H_{s}\right)=S_{i} H_{s}$ and $g\left(H_{i}, S_{s}\right)=H_{i} S_{s}$ (mass action). Figures 1 and 2 present the situation when $R_{0}<1$ and Figures 3 and 4 the case where $R_{0}>1$. In any case, we notice that the curves (black line for the discrete version and blue line for the continuous version) are very close and converge toward the same equilibrium point with respect to the $R_{0}$.

\section{Conclusion}

In order to investigate the dynamics of infectious diseases, many authors analyzed the local and global stability of equilibria of a class of discrete and continuous epidemic models. In this paper, motivated by the fact that discrete models are more appropriate forms than the continuous ones in order to fit the statistical data concerning infectious diseases, we have studied the discrete forward difference version of a schistosomiasis model with general incidence function. From the results obtained in this paper, we see that the backward

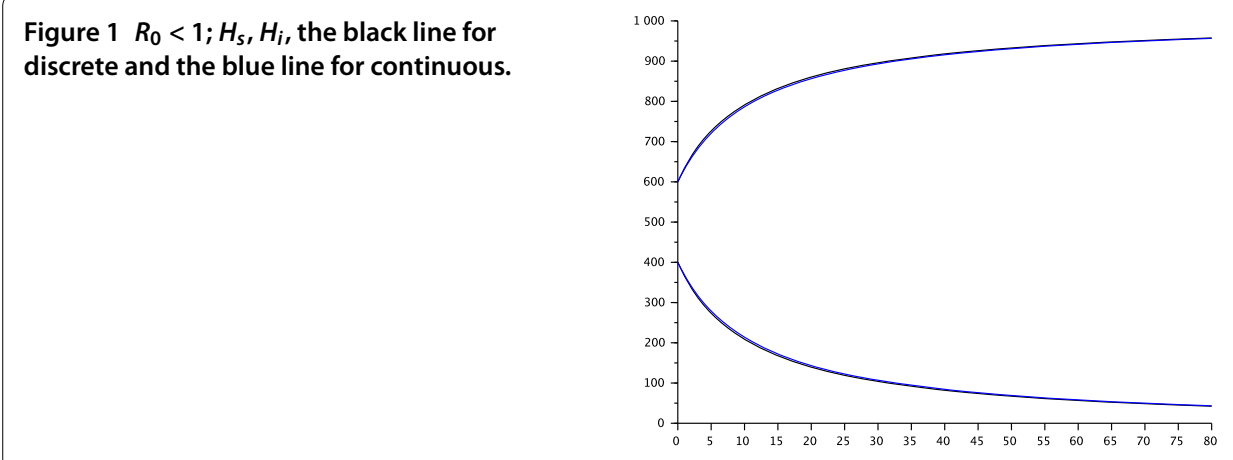




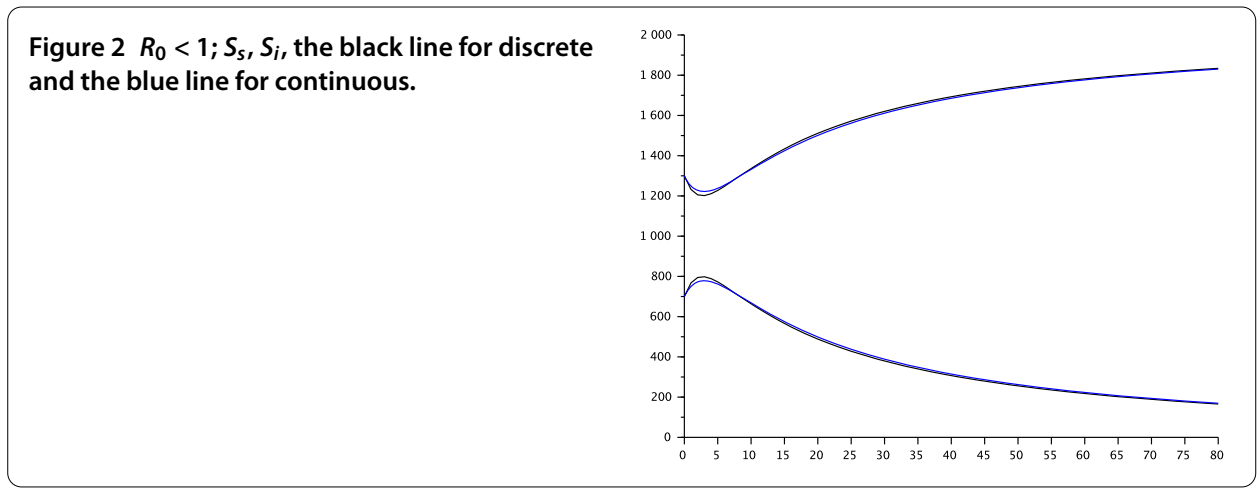

Figure $3 R_{0}>1 ; H_{s}, H_{i}$, the black line for discrete and the blue line for continuous.

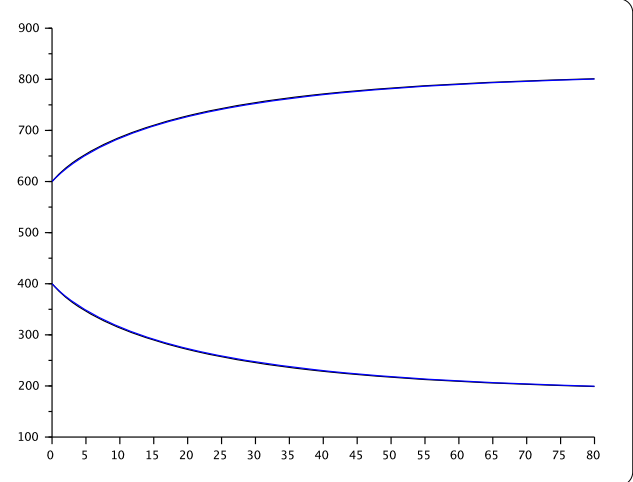

Figure $4 R_{0}>1 ; S_{s}, S_{i}$, the black line for discrete and the blue line for continuous.

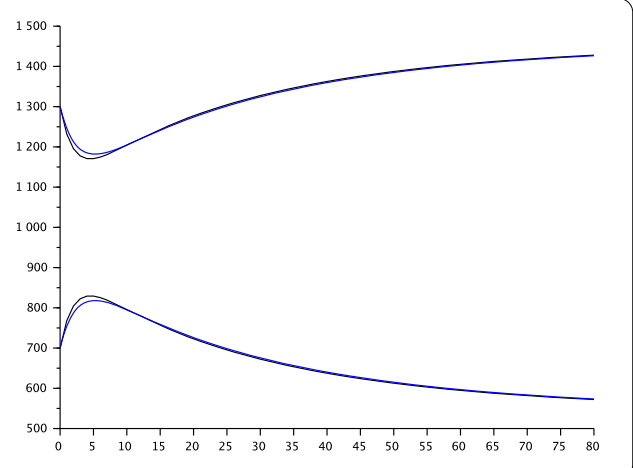

difference scheme, that is, the discrete dynamical model (2), is obtained with excellent dynamical properties for the step size $h=1$ in the local and global stability of equilibria. These properties nearly are the same as the corresponding continuous-time model (1), but the techniques of the computations are quite different. Therefore, we see that the discrete model (2) has more plentiful and complex dynamical behaviors than the continuous model (1). Is this conclusion still right for any step size $h$ ? For future work, we will investigate and try to answer this question. 
Authors' contributions

AG provided the subject, wrote the introduction and the conclusion and verified some calculation. DN conceived the study and computed the equilibria and their local stabilities. DO wrote the mathematical formulas, he used the Lyapunov functional and did all the calculus with the second author. All the authors read and approved the final manuscript.

\section{Author details}

${ }^{1}$ Département de Mathématiques, UFR des Sciences et Techniques, Bobo-Dioulasso, Burkina Faso. ${ }^{2}$ LAboratoire de Mathématique et d'Informatique (LAMI), Université Polytechnique de Bobo-Dioulasso, 01 B.P. 1091, Bobo-Dioulasso, Burkina Faso. ${ }^{3}$ UMI 2019-IRD \& UMMUSCO-UGB, Université Assane Seck de Ziguinchor, B.P. 523, Ziguinchor, Sénégal.

\section{Acknowledgements}

The authors want to thank the anonymous referee for his valuable comments on the paper.

\section{Publisher's Note}

Springer Nature remains neutral with regard to jurisdictional claims in published maps and institutional affiliations.

Received: 3 December 2016 Accepted: 6 April 2017 Published online: 20 April 2017

\section{References}

1. Traore, A, Ouaro, S: Deterministic and stochastic schistosomiasis models with general incidence. Appl. Math. 4, 1682-1693 (2013)

2. WHO: Seventeenth Programme Report of the UNICEF/UNDP/World Bank/WHO Special Program for Research \& Training in Tropical Diseases. http://www.who.int/tdr/publications/publications/pr17.htm

3. World Health Organization: (2012) http://www.who.int/schistosomiasis/en/

4. Steinmann, $P$, et al.: Schistosomiasis and water resources development: systematic review, meta-analysis, and estimates of people at risk. Lancet Infect. Dis. 6, 411-425 (2006)

5. Allen, EJ, Victory, HD: Modelling and simulation of a schistosomiasis infection with biological control. Acta Trop. 87 251-267 (2003)

6. Yang, Y, Xiao, D: A mathematical model with delays for schistosomiasis. Chin. Ann. Math., Ser. B 31(4), $433-446$ (2010)

7. Woolhouse, MEJ: On the application of mathematical models of schistosome transmission dynamic. II. Control. Acta Trop. 50, 189-204 (1992)

8. Izzo, G, Vecchio, A: A discrete time version for models of population dynamics in the presence of an infection. J. Comput. Appl. Math. 210, 210-221 (2007)

9. Izzo, G, Muroya, Y, Vecchio, A: A general discrete time model of population dynamics in the presence of an infection. Discrete Dyn. Nat. Soc. 2009, 143019 (2009)

10. Cao, H, Zhou, Y: The discrete age-structured SEIT model with application to tuberculosis transmission in China. Math. Comput. Model. 55, 385-395 (2010)

11. Castillo-Chavez, C, Yakubu, A-A: Discrete-time S-I-S models with complex dynamics. Nonlinear Anal. 47, 4753-4762 (2001)

12. Enatsu, Y, Nakata, Y, Muroya, Y: Global stability for a class of discrete SIS epidemic models. Math. Biosci. Eng. 7, 347-361 (2010)

13. Chalub, FACC, Souza, MO: Discrete and continuous SIS epidemis models: a unifying approach. Ecol. Complex. 18, 83-95 (2003)

14. Hu, Z, Teng, Z, Zhang, L: Stability and bifurcation analysis in a discrete SIR epidemic model. Math. Comput. Simul. 97, 80-93 (2014)

15. Teng, Z, Wang, Y, Rehim, M: On the backward difference scheme for a class of SIRS epidemic models with nonlinear incidence. J. Comput. Anal. Appl. 20(7), 1268-1289 (2016)

16. Van den Driesche, $P$, Watmough, J: Reproduction numbers and substhreshold endemic equilibria for the compartmental models of disease transmission. Math. Biosci. 180(1-2), 29-48 (2002)

17. Lakshmikantham, V, Leela, S, Martynyuk, AA: Stability Analysis of Nonlinear Systems. Dekker, New York (1989)

18. Lasalle, JP: The Stability of Dynamical Systems. SIAM, Philadelphia (1976)

\section{Submit your manuscript to a SpringerOpen ${ }^{\odot}$ journal and benefit from:}

- Convenient online submission

Rigorous peer review

- Immediate publication on acceptance

Open access: articles freely available online

- High visibility within the field

- Retaining the copyright to your article 